\title{
Pediatric Myelodysplastic Syndrome: Cytomorphological Correlation with Outcome from a Single Tertiary Institution in Oman
}

\author{
Naglaa Fawaz ${ }^{1,2}$, Abdulhakim Al Rawas ${ }^{3}$, Muhammad El Shinawi ${ }^{3}$, Mathew Zachariah ${ }^{3}$, \\ Yasser Wali ${ }^{3}$, Salam Alkindi ${ }^{4}$, Anil Pathare ${ }^{1, ~ *}$ \\ ${ }^{1}$ Department of Hematology, Sultan Qaboos University Hospital, Muscat, Oman \\ ${ }^{2}$ Department of Haematopathology, Suez Canal University, Ismailia, Egypt \\ ${ }^{3}$ Department of Child Health, Sultan Qaboos University Hospital, Muscat, Oman \\ ${ }^{4}$ College of Medicine \& Health Sciences, Sultan Qaboos University, Muscat, Oman
}

Email address:

avp16@hotmail.com (A. Pathare)

${ }^{*}$ Corresponding author

\section{To cite this article:}

Naglaa Fawaz, Abdulhakim Al Rawas, Muhammad El Shinawi, Mathew Zachariah, Yasser Wali, Salam Alkindi, Anil Pathare. Pediatric Myelodysplastic Syndrome: Cytomorphological Correlation with Outcome from a Single Tertiary Institution in Oman. European Journal of Clinical and Biomedical Sciences. Vol. 3, No. 6, 2017, pp. 109-114. doi: 10.11648/j.ejcbs.20170306.12

Received: October 15, 2017; Accepted: October 27, 2017; Published: November 20, 2017

\begin{abstract}
Pediatric MDS is currently classified into three distinct groups namely MDS, Juvenile myelomonocytic leukemia (JMML) and Down Syndrome-associated leukemias (DSAL). The aim of this study was to evaluate pediatric MDS patients using the WHO 2008 morphological classification with the clinical outcome. In this retrospective single centre study, 19 pediatric MDS patients (median age 6 years; range 3 months -16 years) were analyzed for their initial presentation, type of progression, leukemic transformation and overall survival as well as MDS related cytogenetic abnormalities. The median follow-up was 26.5 months. The most common single presentation of childhood MDS was RCC (26\%). Leukemic transformation was seen in almost half of this cohort but was completely restricted to the DSAL and RAEB-T subgroups only. The median survival in the MDS group was 50 months, whereas for the entire cohort it was 35 months. RCC cohort showed the best survival with the median of 53.75 months, whereas the Down-related disease cohort showed the worst survival estimates with a median of only 11.25 months. Multivariate analysis showed that gender, platelet count, HbF, bone marrow cellularity, bone marrow blast percentage contributed significantly to the prognosis and survival. The study shows that cytogenetics, including monosomy 7 did not influence the outcome or the overall survival of childhood MDS. We identified that thrombocytopenia and BM blasts $>5 \%$ were associated with a poor survival in this childhood MDS cohort.
\end{abstract}

Keywords: Pediatric, MDS, Cytopenia, Myelodysplasia, Monosomy 7, Down's Syndrome

\section{Introduction}

Although MDS is one of the most common hematopoietic disorders in the elderly with a median age of 70 years at diagnosis, it is less frequently seen in pediatrics and is known to occur both as de novo and secondary forms. [1, 2] Presence of bone marrow failure, which manifests itself as cytopenia is an important indication for bone marrow investigation is this setting. The causes of cytopenia are varied and differ considerably between childhood and adulthood, as inherited bone marrow failure syndromes [IBMFS] are important causes of bone marrow failure in the pediatric age group but play only a minor role in adulthood. $[2,3]$ Furthermore, inherited bone marrow failure syndromes have a varied propensity to develop secondary MDS. Consequently, the diagnosis of pediatric MDS is really challenging particularly in a country like Oman with high rate of consanguinity and inherited hematological and nonhematological diseases. Moreover, dysplasia is frequently observed in non-MDS conditions including metabolic 
disorders, serious infections associated with congenital immune deficiency syndromes, hemophagocytic lymphohistiocytosis, in addition to inherited bone marrow failure syndromes (IBMFS). [2-8] Therefore, MDS can mimick a large variety of hematological and nonhematological diseases and there is considerable difficulty in assigning the overlapping morphological features seen in bone marrow failure syndromes with MDS.

MDS is a clonal disorder and the current pediatric classification separates these clinical entities into three distinct groups namely MDS, Juvenile myelomonocytic leukemia (JMML) and Down Syndrome-associated leukemias (DSAL). MDS can also be primary or secondary, based on the combination of pathognomic findings in peripheral blood smears, bone marrow histology, cytogenetics, and clinical examination. The classification and diagnostic criteria for MDS have evolved since the initial French-American-British classification of MDS in 1982. [9] Using cytogenetic abnormalities and number of bone marrow blast, the World Health Organization (WHO) in 2001 classified several subtypes of MDS, albeit in adults. [10] It was only in year 2003 that a pediatric modification to the WHO Classification was proposed categorizing these disorders into 3 distinct groups namely MDS, JMML and DSAL. [11] The revised WHO classification in 2008 confirmed these three entities and defined the precise criteria for a pediatric type of MDS called refractory cytopenia of childhood (RCC). This group was defined as children with $<2 \%$ blast in peripheral blood, $<5 \%$ blast in the bone marrow and persistent cytopenia with dysplasia in at least two cell lineages. [12] Thus, subjects with $2 \%-19 \%$ blasts in the blood or $5-19 \%$ in the bone marrow indicating refractory anemia with excess blasts (RAEB) - and with 20\%-29\% blasts in the peripheral blood and the bone marrow were classified as RAEB-T. [13]

The aim of this of study is to assess pediatric MDS patients, considering their initial presentation, morphology in peripheral blood and bone marrow, type of progression and overall survival as well as the results of cytogenetic analysis in the light of the current WHO classification from a single tertiary institution in Oman. The diagnosis of pediatric MDS was based on cytomorphological and laboratory criteria as defined in current literature. [12, 13]

\section{Method}

\subsection{Patients}

In this retrospective study, we assessed 19 pediatric MDS patients from a single tertiary institution in Oman. For the distinction between primary and secondary disease, any known predisposing condition was taken into consideration in each case e.g. prior medications (e.g. antiepileptics), chemotherapy and /or radiotherapy, a previously well-defined bone marrow failure syndrome or a pre-existing bone marrow disorders. Metabolic diseases, rheumatic diseases and nutritional vitamin $\mathrm{B}_{12}$ and folate deficiency, were excluded as well as hemoglobinopathies because of the high prevalence in Oman. Virus-specific serology for exclusion of EBV, CMV, HHV6, Herpes simplex and Parvovirus B19 infection were also performed.

\subsection{Materials and Methods}

\subsubsection{Cytomorphological Analysis}

Peripheral blood (PB) and bone marrow (BM) morphology was evaluated on Wright stained preparations and was based upon a minimum of 200 nucleated cells for PB and at least 300 for BM. Dyserythropoiesis manifested principally nuclear budding, inter-nuclear bridging and karyorrhexis, multinuclearity and megaloblastic changes. Dysgranulopoiesis was characterized primarily by nuclear hypolobation (pseudo Pelger-Huet), hypersegmentation and hypogranularity. Megakaryocyte dysplasia was characterized by hypolobated micromegakaryocyte, non-lobulated nuclei in megakaryocytes of all sizes and multiple, widely-separated nuclei. Quantitative and qualitative abnormalities of all three haemopoietic cell lineages on proliferation and maturation process were evaluated, and at least a two-cell lineage dysplasia for a minimum of $10 \%$ of cells was required to qualify as dysplasia. The presence of sideroblasts on BM specimens was assessed by Perl's stain. Bone marrow biopsies were examined with particular attention to overall cellularity, evaluated according to conventional microscopic standards on H-E sections using semi-quantitative evaluation and adjusted to each patient's age. Reticulin fibers or fibrosis were identified by cytochemistry.

\subsubsection{Chromosomal Analysis}

Bone marrow samples were cultured using Marrow Max culture media (cat. \#12260e022; Gibco, Grand Island, NY) for 24 and $48 \mathrm{~h}$ using standard protocols. Chromosome preparation was done by standard short-term culture (24 and $48 \mathrm{~h}$ ) techniques using RPMI-1640 marrow max medium (GIBCO cat. \# 12260e022) incubated at $37^{\circ} \mathrm{C}$. Karyomaxe Colcemid solution (final concentration $0.05 \mathrm{mg} / \mathrm{mL}$ ) was added for the final $30 \mathrm{~min}$ of culture. Chromosome analysis was performed on GTG-banded preparations using the Applied Imaging Cytovision Automated Karyotyping System. International System for Human Cytogenetic Nomenclature (ISCN) criteria (2005) was used for assessing the presence of clones with numerical and structural abnormalities. [14] The chromosome breakage analysis on PB lymphocytes with mitomycin was used to evaluate the diagnosis of Fanconi anaemia.

\subsubsection{Statistical Analysis}

Statistical Analysis was performed using STATA ver. 10.0 (StataCorp, College Station, TX, USA). Univariate and Multivariate analysis was performed using the Kaplan-Meier curves for all predictors to provide an insight into the survival function of each predictor and whether or not the subgroups analyzed are proportional or not. The Univariate Cox proportional hazard regression was used for continuous variables, whereas, the log-rank test of equality was used for 
categorical variables. If the predictor had a higher p-value than 0.25 in the univariate analysis, it was excluded in building the multivariate model as it is quite unlikely that it would contribute significantly to the multivariate model that includes other predictors. A p-value of $<0.05$ was considered as statistically significant.

\section{Result}

The demographic and laboratory findings as well as the WHO 2008 subtypes, cytogenetic abnormalities and the clinical outcome in this patient cohort is shown in Table 1. The median age was 6 years, with a range from 3 months to
16 years. The median follow-up was of 26.5 months with the majority (94\%) of patients presenting with two or three lineage cytopenias with dysplasia. The most common single presentation of childhood MDS was RCC (26\%) although overall Downs-related disorders with the single most frequent condition in this cohort. Leukemic transformation was seen in almost half of this cohort but was entirely restricted to the Downs-related and RAEB-T subgroups only. Of the 4 patients $(21 \%)$ who died, three deaths were due to sepsis and one case died following fatal intracranial bleed due to intractable thrombocytopenia. Surprisingly none of the deaths occurred in patients with poor cytogenetic abnormalities.

Table 1. Patient characteristics.

\begin{tabular}{|c|c|}
\hline Characteristics & Number \\
\hline Total Patients, $n$ & 19 \\
\hline Male/Female & $10 / 9$ \\
\hline Mean age, years & 8.2 \\
\hline Median Age, years & 6 \\
\hline Range, years & $0.3-16$ \\
\hline \multicolumn{2}{|l|}{ Follow up } \\
\hline Median (Range), months & $26.5(1-69)$ \\
\hline \multicolumn{2}{|l|}{ Peripheral Blood Count } \\
\hline Median hemoglobin (g/dl)(range) & $8.8(6-11.5)$ \\
\hline Median ANC $\left(\mathrm{X} 10^{9} / \mathrm{L}\right)$ (range) & $1.04(0.1-3.4)$ \\
\hline Median Platelets $\left(\mathrm{X} 10^{9} / \mathrm{L}\right)($ range $)$ & $47(3-129)$ \\
\hline \multicolumn{2}{|l|}{ Cytopenia, $n(\%)$} \\
\hline Single lineage & $1(6)$ \\
\hline Bilineage & $9(47)$ \\
\hline Trilineage & $9(47)$ \\
\hline \multicolumn{2}{|l|}{ Bone Marrow Blast $\%, n(\%)$} \\
\hline$<5$ & $8(42)$ \\
\hline 5-10 & $4(21)$ \\
\hline$>10$ & $7(37)$ \\
\hline \multicolumn{2}{|l|}{ Therapy, $n(\%)$} \\
\hline Supportive only & $7(37)$ \\
\hline Chemotherapy & $7(37)$ \\
\hline Bone Marrow Transplantation & $5(26)$ \\
\hline \multicolumn{2}{|l|}{ WHO 2008 subtype $n(\%)$} \\
\hline $\mathrm{RCC}$ & $5(26)$ \\
\hline RAEB & $3(15)$ \\
\hline RAEB-T & $2(11)$ \\
\hline JMML & $2(11)$ \\
\hline Downs-related & $7(37)$ \\
\hline \multicolumn{2}{|l|}{ Leukemic Transformation } \\
\hline Yes, $n(\%)$ & $10(52)$ \\
\hline No, $n(\%)$ & $9(48)$ \\
\hline \multicolumn{2}{|l|}{ Status, Dead } \\
\hline Yes, n(\%) & $4(21)$ \\
\hline No, $n(\%)$ & $15(79)$ \\
\hline \multicolumn{2}{|l|}{ Cause of Death } \\
\hline Sepsis, $n(\%)$ & $3(75)$ \\
\hline IC Bleed, n(\%) & $1(25)$ \\
\hline \multicolumn{2}{|l|}{ Cytogenetics } \\
\hline Normal & $6(33)$ \\
\hline Single abnormality $(-7,+21,+8)$ & $8(44)$ \\
\hline Complex abnormalities $(+21$ with del16, Del10, $\operatorname{der}(8))$ & $4(22)$ \\
\hline
\end{tabular}

Censoring and non-normality in survival analysis data makes it difficult to use the traditional statistical analysis models like multiple linear regression. Hence, survival analysis was carried out by using the Kaplan-Meier survival estimates [Figures 1a-d]. Overall survival was defined as the time between diagnosis of MDS and death or its likelihood. The median survival in the MDS group was 50 months [Figure 1c], whereas for the entire cohort it was 35 months [Figure 1a]. RCC cohort showed the best survival with the median of 53.75 months, whereas the Down-related disease 
cohort showed the worst survival estimates with a median of

only 11.25 months. [Figure 1b]
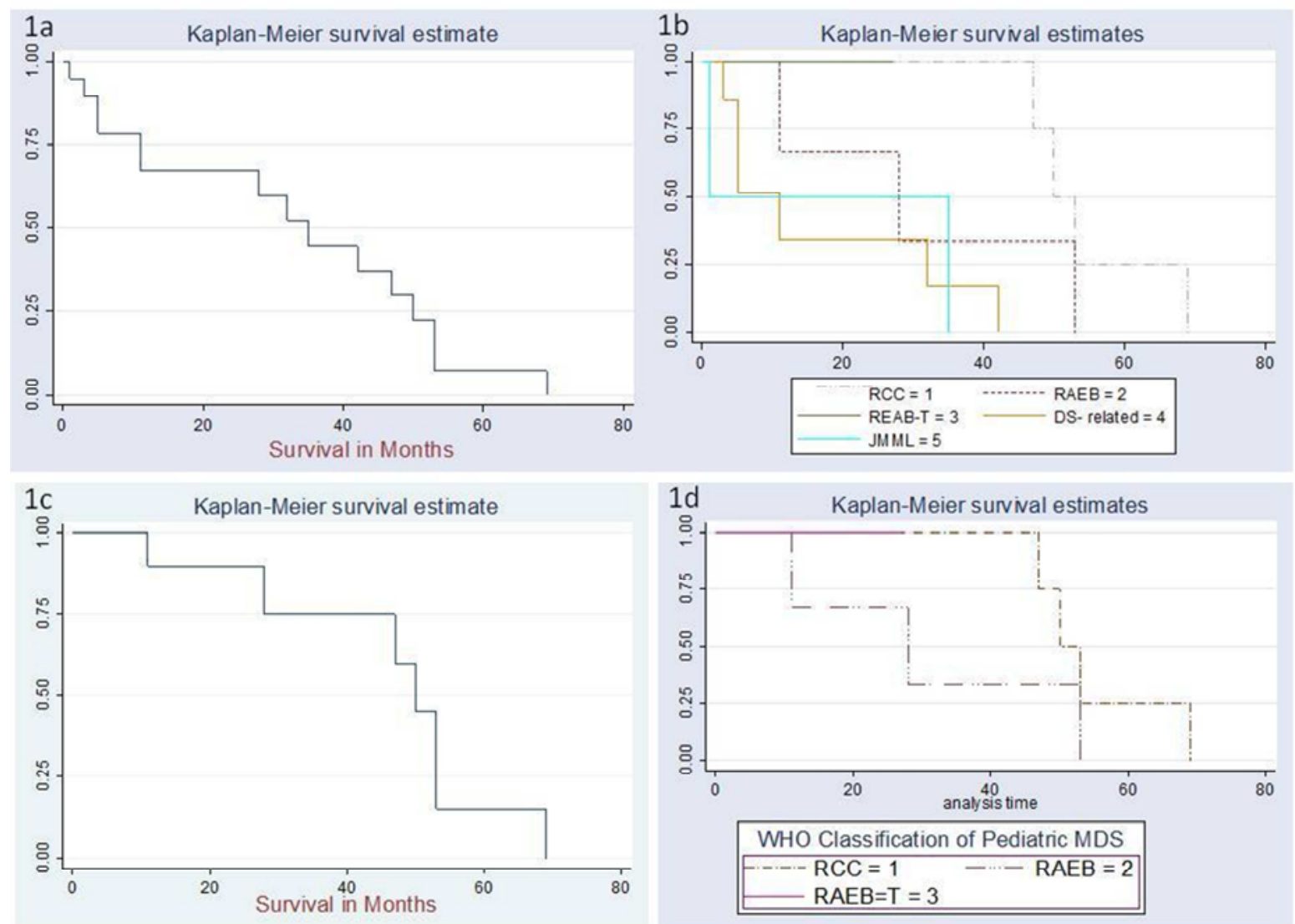

Figures 1. $[a-d]$.

1a: Kaplan-Meier survival estimate for the entire cohort [n=19] with a median survival of 35 months.

1b: Kaplan-Meier survival estimates for the entire cohort [ $\mathrm{n}=19]$ with a median survival for RCC, RAEB, JMML and DS-related disorder respectively being $53.75,27.5,35$, and 11.25 months. Since both the RAEB-T patient in this cohort had died, hence no survival estimate could be obtained.

1c: Kaplan-Meier survival estimate for the MDS cohort [ $\mathrm{n}=10]$ with a median survival of 50 months.

1d: Kaplan-Meier survival estimates for the MDS cohort [n=10] with a median survival for RCC and RAEB, respectively being 53.75 and 27.5 months. Since both the RAEB-T patient in this cohort had died, hence no survival estimate could be obtained.

Tables 2 \& 3 show the results of univariate and multivariate analysis using the Cox proportional hazards regression and the logrank test of equality, respectively. The initial univariate analysis showed that the age, WBC count, $\mathrm{BM}$ blast $\%$ and the number of cytopenia were significantly associated with poor prognosis. However, multivariate analysis showed that gender, platelet count, $\mathrm{HbF}$, bone marrow cellularity, bone marrow blast $\%$ and the nature of therapy contributed significantly to the prognosis and survival thereafter.

Table 2. Univariate analysis of covariates.

\begin{tabular}{|c|c|c|c|c|c|}
\hline Predictor & Coefficient & $+\mathbf{S E}$ & $\mathbf{P}$ & $95 \%$ & Confidence Interval \\
\hline Age & -0.161 & 0.071 & 0.025 & -0.302 & -0.0204 \\
\hline Gender & 1.161 & 0.696 & 0.095 & -0.202 & 2.526 \\
\hline $\mathrm{Hb}$ & -0.011 & 0.189 & 0.952 & -0.382 & 0.359 \\
\hline WBC & 1.556 & 0.065 & 0.018 & 0.027 & -0.284 \\
\hline ANC & 0.350 & 0.311 & 0.261 & -0.260 & 0.961 \\
\hline Platelets & 0.008 & 0.006 & 0.211 & -0.005 & 0.022 \\
\hline $\mathrm{MCV}$ & -0.002 & 0.031 & 0.941 & -0.064 & -0.060 \\
\hline BM Cellularity & 0.238 & 0.188 & 0.206 & -0.131 & 0.609 \\
\hline Leukemic Transformation & 0.363 & 0.553 & 0.512 & -0.721 & 1.448 \\
\hline BM Blast $\%$ & 0.032 & 0.016 & 0.041 & 0.001 & 0.064 \\
\hline No. of Cytopenias & -2.186 & 0.816 & 0.007 & -3.786 & -0.585 \\
\hline Therapy & -0.571 & 0.355 & 0.108 & -1.267 & 0.125 \\
\hline Cytogenetic Abnormalities & 0.324 & 0.421 & 0.442 & -0.502 & 1.150 \\
\hline
\end{tabular}


Table 3. Multivariate analysis model.

\begin{tabular}{llllll}
\hline Predictor & Coefficient & +SE & P & 95\% & Confidence Interval \\
\hline Gender & -16.03 & 8.130 & 0.049 & -31.96 & -0.098 \\
Platelets & -0.045 & 0.022 & 0.042 & -0.089 & -0.001 \\
HbF & 0.664 & 0.250 & 0.008 & 0.173 & 1.154 \\
BM Cellularity & 11.39 & 3.169 & 0.001 & 5.181 & 0.007 \\
BM Blast\% & 0.410 & 0.205 & 0.046 & -17.60 & 0.812 \\
Therapy & -9.604 & 4.336 & 0.027 & -1.10 & \\
\hline
\end{tabular}

\section{Discussion}

\subsection{Clinical and Laboratory Abnormalities}

Nineteen patients, who were less than 18 years of age, were included in this study from a single center in Oman and their data was retrospectively analyzed [Table 1]. These children usually presented with symptoms related to cytopenia, such as anemia, infection, and bleeding tendency. We have consistently used the WHO 2008 morphological classification amongst our patients as a pivotal descriptive tool throughout the period of our study. However, we encountered several difficulties in the differential diagnosis of childhood MDS, owing to the fact that similar morphological findings may be non-specific and may be found in various other conditions, such as infections, acquired aplastic anemia, hemophagocytic lymphohistiocytic syndrome, non-classic phenotype of $\mathrm{PNH}$, congenital dyserythropietic anemia, dyskeratosis congenita, severe congenital neutropenia, and other IBMFS. But by carefully applying the current WHO classification criteria for determining MDS in children and by following these patients closely, correct diagnosis could be established. All children were diagnosed between 2008 and 2013. An observation time longer than 4 months from diagnosis was required for the inclusion in the study cohort. We incorporated patients who had received haemopoietic stem cell transplantation [HSCT] in the study cohort as we realized that the study would be revealing if transplanted patients were included. Moreover, early HSCT is an established treatment of choice in childhood MDS because of the good overall outcome of such patients.

\subsection{Bone Marrow Cellularity}

According to our study data, it appears that hypocellularity of the bone marrow is frequent among children at diagnosis, while normocellular or hypercellular marrow is often restricted to JMML and DSAL. This finding was also observed by other investigators. $[15,16]$ Hence the diagnostic challenge is narrowed to differentiating MDS with low blast counts from aplastic anemia (AA) and MDS with excess blast from AML. Since RCC was the most common presentation in our childhood MDS cohort, MDS-related cytogenetic abnormality, when present, was of considerable importance in helping to differentiate between RCC and AA or IBMFS. Conversely, for patients with equivocal cytogenetics and bone marrow blast counts between $20 \%$ and $29 \%$, AML was a major differential diagnosis of MDS.

\subsection{Cytogenetics}

A bone marrow blast count of $<20 \%$ may indicates MDS, although cytogenetics rather than a cutoff blast count value was of considerable importance. The presence of monosomy 7 strongly suggests MDS [17, 18] and actually $44 \%$ of this patient cohort had monosomy 7 either alone or with additional cytogenetics aberrations. Triosmies of chromosomes 21 and 8 were the second most common cytogenetics abnormality amongst this patient cohort, whereas, the 5q- abnormality, commonly found in adults, was completely absent in our pediatric patient cohort. [19] The rarity of this cytogenetic change in children has been confirmed in other series also. $[15,16]$

\subsection{Leukemic Transformation}

Leukemic transformation was seen in almost half of our patients, but was only restricted to the Downs- related and RAEB-T subgroups. In our study, univariate analysis, did not find cytogenetic abnormality as with poor prognosis $(p=0.44)$. Moreover, although chromosomal abnormality is linked to prognosis, none of the deaths in this patient cohort occurred in subjects with poor cytogenetic abnormalities. Furthermore, monsomy 7 , the most common abnormality in children was not associated with poor prognosis, unlike in adults. The lack of monosomy 7 as an unfavorable feature in MDS has also been observed in previous studies. [17, 18] However, few studies have suggested that children with monosomy 7 progress earlier to AML. [20]

\subsection{Overall Survival}

This study documented superior survival in MDS patients with RCC and marrow blasts $<5 \%$ with the median survival of 53.75 months, whereas, in the Down-related disease, usually classified as RAEB or RAEB-T, it showed the worst survival with a median of only 11.25 months [Figure 1]. Similar findings also observed by others. [20, 21] Bone marrow cellularity and blast percentage significantly influenced survival in both univariate analysis, using the Cox proportional hazards regression and the multivariate regression model. Additionally, multivariate analysis of variables influencing the probability of overall survival also demonstrated that thrombocytopenia and elevated $\mathrm{HbF}$ level as poor prognostic markers.

\section{Conclusion}

In summary, the study showed that cytogenetics, including 
monosomy 7 did not influence the outcome or the overall survival of childhood MDS. We identified thrombocytopenia and BM blasts $>5 \%$ as being associated with a poor survival in childhood MDS. HSCT was seen to be usually associated with good results especially if it can be performed early before progression or other complications have occurred.

\section{Conflict of Interest}

The authors declare that they have no competing interests.

\section{References}

[1] Williamson PJ, Kruger AR, Reynolds PJ, Hamblin TJ, Oscier DG. Establishing the incidence of myelodysplastic syndrome. $\mathrm{Br} \quad \mathrm{J} \quad$ Haematol, . 1994; 87:743-745. https://doi.org/10.1111/j.1365-2141.1994.tb06733.x

[2] Alter BP, Giri N, Savage SA, et al. Malignancies and survival patterns in the National Cancer Institute inherited bone marrow failure syndromes cohort study. Br J Haematol. 2010;150:179-188. https://doi.org/10.1111/j.13652141.2010.08212.x

[3] Glaubach T, Robinson LJ, Corey SJ. Pediatric myelodysplastic syndromes: they do exist! J Pediatr Hematol Oncol. 2014; 36:1-7. https://doi.org/10.1097/mph.0000000000000046

[4] Hasle H, Wadsworth LD, Massing BG, et al. A population based study of childhood myelodysplastic syndrome in British Columbia, Canada. Br J Haematol. 1999;106:1027-1032. https://doi.org/10.1046/j.1365-2141.1999.01645.x

[5] Passmore SJ, Chessells JM, Kempski H, et al. Paediatric myelodysplastic syndromes and juvenile myelomonocytic leukaemia in the UK: a population-based study of incidence and survival. $\mathrm{Br} \mathrm{J}$ Haematol. 2003;121:758-767. https://doi.org/10.1046/j.1365-2141.2003.04361.x

[6] Bader-Meunier B, Mielot F, Tchernia G, et al. Myelodysplastic syndromes in childhood: report of 49 patients from a French multicenter study. Br J Haematol 1996; 92: 344-50. https://doi.org/10.1046/j.1365-2141.0000.d01-1480.x

[7] Sasaki H, Manabe A, Kojima S, et al. Myelodysplastic syndrome in childhood: a retrospective study of 189 patients in Japan. Leukemia. 2001;15:1713-1720. https://doi.org/10.1038/sj.leu.2402271

[8] Luna-Fineman S, Shannon KM, Atwater SK, et al. Myelodysplastic and myeloproliferative disorders of childhood: a study of 167 patients. Blood. 1999;93:459-466.

[9] Bennett JM, Catovsky D, Daniel MT, et al. Proposals for the classification of the myelodysplastic syndromes. $\mathrm{Br} \mathrm{J}$
Haematol. 1982; 51:189-199 https://doi.org/10.1111/j.13652141.1982.tb02771.x

[10] Jaffe JS, Harris NL, Stein H, Vardiman JW, eds. World Health Organization Classification of Tumors. Pathology and Genetics of Tumors of Hematopoietic and Lymphoid Tissues. 3rd ed. Lyon, France: IARC Press; 2001.

[11] Hasle H, Niemeyer CM, Chessells JM, et al. A pediatric approach to the WHO classification of myelodysplastic and myeloproliferative diseases. Leukemia. 2003; 17:277-282. https://doi.org/10.1038/sj.leu.2402765

[12] Swerdlow SH, Campo E, Harris NL, et al, eds. WHO Classification of Tumours of Haematopoietic and Lymphoid Tissues. 4th ed. Lyon, France: IARC Press; 2008.

[13] Vardiman JW, Thiele J, Arber DA, et al. The 2008 revision of the World Health Organization (WHO) classification of myeloid neoplasms and acute leukemia: rationale and important changes. Blood. 2009;114:937-951. https://doi.org/10.1182/blood-2009-03-209262

[14] Mitelman F, editor. ISCN (1995): An international system for human cytogenetic nomenclature. Basel: S Karger; 1995.

[15] Niemeyer CM, Baumann I. Myelodysplastic syndrome in children and adolescents. Semin Hematol. 2008;45:60-70. https://doi.org/10.1053/j.seminhematol.2007.10.006

[16] Pitman SD, Victorio A, Rowsell E, et al. 5qsyndrome in a child with slowly progressive pancytopenia: a case report and review of the literature. J Pediatr Hematol Oncol. 2006; 28: $115-119$.

https://doi.org/10.1097/01.mph.0000210410.48877.15

[17] Kardous G, Baumann I, Passmore SJ, et al. Refractory anemia in childhood: a retrospective analysis of 67 patients with particular reference to monosomy 7. Blood. 2003;102:19972003. https://doi.org/10.1182/blood-2002-11-3444

[18] Gohring G, Michalova K, BeverlooHB, et al. Complex karyotype newly defined: the strongest prognostic factor in advanced childhood myelodysplastic syndrome. Blood. 2010;116:3766-3769. https://doi.org/10.1182/blood-2010-04280313

[19] Boultwood J, Lewis S, Wainscoat JS. The 5q- syndrome. Blood. 1994; 84:3253-3260.

[20] Delhommeau F, Dupont S, Della Valle V, et al. Mutation in TET2 in myeloid cancers. N Engl J Med. 2009 May 28;360:2289-2301. https://doi.org/10.1056/nejmoa0810069

[21] Verburgh E, Achten R, Louw VJ, et al. A new disease categorization of low-grade myelodysplastic syndromes based on the expression of cytopenia and dysplasia in one versus more than one lineage improves on the WHO classification. Leukemia. 2007;21:668-677. https://doi.org/10.1038/sj.leu.2404564 